\title{
The Initial Implementation of the Sentinel Lymph Node Biopsy (SLNB) for Breast Cancer Management in Malta
}

\author{
Camilleri Gail $^{1}$, Borg Grima Karen ${ }^{2}$, Zarb Francis ${ }^{2}$ \\ ${ }^{1}$ Medical Imaging Department, Mater Dei Hospital, Msida, Malta; ${ }^{2}$ Department of Radiography, Faculty of Health Sciences, Univer- \\ sity of Malta, Msida, Malta. \\ Email: gail.camilleri@gov.mt, karen.borg-grima@um.edu.mt, francis.zarb@um.edu.mt
}

Received March 16 ${ }^{\text {th }}, 2013$; revised April 17 ${ }^{\text {th }}, 2013$; accepted April 25 ${ }^{\text {th }}, 2013$

Copyright (c) 2013 Camilleri Gail et al. This is an open access article distributed under the Creative Commons Attribution License, which permits unrestricted use, distribution, and reproduction in any medium, provided the original work is properly cited.

\begin{abstract}
Over the past two decades, the sentinel lymph node biopsy (SLNB) based on sentinel node (SN) being the first lymph node that harbors metastases, revolutionized breast cancer management. SLNB presents much less morbidity when compared to radical axillary lymph node dissection (ALND) where all nodes are dissected irrespective of their metastatic involvement. The purpose of this study was to evaluate the effectiveness of SLNB by investigating whether the histological characteristics of the SNs identified using scintigraphy are predictive of the histological characteristics of the ALN basin. Methods: Fifty-five female breast cancer patients underwent lymphoscintigraphy and SLNB followed by ALND. The histological status of the SN/s was correlated to the histological status of the ALNs to determine whether the SN accurately stages the ALNs in breast cancer. Results: During surgery, SNs were successfully isolated in 52 out of 55 cases (94.5\%) (range, 0 to 9). No SNs were identified in 3 cases (5.5\%). Results demonstrate a significant association $(\mathrm{p}=0.05)$ between the metastatic status of SNs and the corresponding ALNs in 42 out of 52 patients (80.8\%), but with a high false-negative rate (FNR) of 37.5\%. Conclusion: The findings of this study show that the sentinel node concept provides the benefits of SLNB in the majority of instances. However, further work is required in reducing the FNR. Once the effectiveness of SLNB as a staging technique is locally established, the need of ALND in SN-negative patients would be limited, thus improving the quality of life of Maltese breast cancer patients.
\end{abstract}

Keywords: Breast Cancer; Sentinel Node; Lymphoscintigraphy; Sentinel Lymph node Biopsy; Axillary Lymph Node Dissection

\section{Introduction}

Over the past decades, axillary lymph node dissection (ALND) was universally accepted as the most accurate prognostic tool available in evaluating axillary nodal status and hence breast cancer staging [1-3]. The minimally invasive technique of the sentinel lymph node biopsy (SLNB) marked an important epoch in the history of breast cancer management [4,5]. The major drawback in performing routine ALND is associated with longterm complications including post-operative lymphedema, paresthesia, neuropathy, seroma formation, chronic shoulder pain, joint disruption, weakness and reduced mobility [2,3,6-9]. The SLNB can therefore be used to select breast cancer patients needing total dissection [10], catering for an improved quality of life and shorter hospital stay. The SLNB procedure does not exclude side effects, but risks are substantially reduced in comparison to the more invasive ALND [11-13].
Due to the steadily increasing rates in breast cancer in Malta, ranking among the highest in Europe at 34.4 per 100,000 million [14], staging procedures with fewer complications were being sought. The SLNB was thus locally introduced in May 2009. The SLNB is based on the concept of the orderly progression of lymph node metastases, where only one or few lymph nodes (termed the sentinel node/s, (SN/s)) are the first to be reached by metastasizing cells from tumor dissemination $[15,16]$. All SNs need to be surgically harvested during SLNB to stage the disease, thus minimizing patient morbidity. It appears that the presence of more than $1 \mathrm{SN}$ is a common finding in the context of breast cancer as multiple lymphatic channels may emanate from the same lesion [1719]. The lymphatic drainage of the breast (and hence the SN) is confined to the ipsilateral axilla in $75 \%$ to $95 \%$ of cases, although variation in the lymphatic flow exists [13,20-22]. 
It is well documented that there is a relationship between the histological findings of the sentinel and axillary nodes, in that the SN represents the metastatic status of the axillary nodal basin. From this perspective, a tumor-negative SN equates to tumor-negative ALNs, similarly a tumor-positive SN indicates that breast cancer has metastasized to the ALNs $[3,9,18]$. Nevertheless, exceptions exist in a minority of anomalous patients where the SN does not truly reflect the status of the axilla in terms of metastases, as the tumor may bypass the SN. This is a serious situation referred to as the false-negative rate (FNR) of SLNB, which accounts for $0 \%$ to $19 \%$ of biopsies where ALNs positive for tumor are present beyond an undetected SN [8,23-25].

The purpose of this study was to evaluate whether the histological characteristics of SNs identified on lymphoschintigraphy and harvested during SLNB, predict the histological characteristics of ALNs in terms of metastases. This was achieved by correlating the histological status of the SN/s to the histological status of the ALNs.

\section{Materials and Methods}

The study was approved by the University of Malta Research and Ethics Committee (UREC ref. no: 077/2010). Approval to perform the research was also sought and obtained from the Imaging Department, Pathology Department and Data Protection Office of the hospital from where the data was collected.

Between May 2009 and December 2010, data for 55 consecutive pre- and post-menopausal female breast cancer patients aged between 27 to 81 years (mean 58.35) was considered in this retrospective study. Exclusion criteria were: pregnancy, lactation, previous surgery at the affected breast, clinically suspected metastatic involvement of the axilla (palpable axillary nodal metastases) or cases of multifocal and multicentric carcinomas of the breast. In the latter more than one tumor is present in the breast quadrants and consequently commonly present with multiple drainage patterns involving more than 1 SN [26]. No males were included in this series.

All candidates underwent lymphoscintigraphy and were scheduled to receive SLNB and total or partial back-up ALND at the general hospital in Malta on the same day or on the day following imaging. Patients were initially seen at the Nuclear Medicine Unit for the cutaneous (sub dermal) peri-areolar administration of the radiopharmaceutical (15-25MBq of Tc-99m nanocolloid) within the tumor-bearing quadrant. There was no reported adverse or clinically detectable pharmacologic effect in any of the 55 subjects.

Pre-operative lymphoscintigraphic imaging was performed between 1 to 2 hours following radiopharmaceutical administration. Anterior, anterior oblique and lateral erect lymphoscintigrams were obtained in static views using a low-energy high-resolution collimator. In order to aid the localization of the SN during surgery, radiographers silhouetted the patient's breast areas using a radioactive cobalt-67 flat flood wire source on the anterior and lateral views whilst the anterior oblique view was used to locate the sentinel nodes with a cobalt-67 pen source and marked the skin overlying the SN/s using inedible ink [27]. The number and location of the SN/s were recorded. The criteria for a successful breast lymphoscintigram consisted of the demonstration of the injection site together with the visualization of at least 1 $\mathrm{SN}$ [3].

In order to facilitate intra-operative identification of the SN/s during surgery, a hand-held gamma-probe was utilized at all times and occasionally complimented by the relatively safe methylene blue dye technique. The blue dye is subdermally administered a few minutes prior to the commencement of the biopsy and allows the visual confirmation of lymphatic pathways from the tumor site to the SN/s $[28,29]$. During surgical exploration the SN was defined as the "hot" node, registering a radiation count tenfold the normal background on the gamma probe and/or the blue stained node with the associated blue dye technique [5]. A successful SLNB is defined as the identification and excision of at least $1 \mathrm{SN}$.

During SLNB, lymph nodes were divided into 2 groups: the radioactive nodes and/or the visually stained lymph node/s identified as SNs (specimen 1) and the remainder of lymph nodes excised through the axillary dissection (specimen 2). These 2 specimens, along with the breast specimen of the primary tumor were labeled and subjected to pathological analysis.

The SNs and ALNs of each patient were placed in $10 \%$ buffered formalin (pH7) to ensure proper fixation. Sectioned at $2 \mathrm{~mm}$ intervals, the entire SNs were submitted for evaluation while the remaining ALNs were semi-bisected. These specimens were processed for tissue shaping and impregnation. Subsequently, all nodes were embedded in paraffin wax according to standard procedures. For each specimen, initial sections were cut and de-waxed by hematoxylin and eosin (H \& E) staining. The cover slips were microscopically evaluated by the consultant pathologist who reported any metastatic involvement of the lymph nodes.

The patients' age; number of visualized SNs during lymphoscintigraphy; corresponding number of identified SNs and ALNs during SLNB and their histological evaluation in terms of metastatic deposit, was recorded retrospectively by looking up lymphoscintigraphy reports and histology results. Diagnostic accuracy was calculated by the positive predictive values (PPV) and the falsenegative rate (FNR) of the sentinel node histology compared to axillary node status (as the reference standard) [30] and evaluating the sensitivity and specificity of the 
procedure. The FNR is calculated by the number of falsenegative procedures divided by the sum of the true-positives (TP) and false-negatives (FN) $[6,31,32]$. Sensitivity is calculated by the number of TP procedures divided by the sum of the TP and FN. On the other hand, specificity is the calculated by the number of true-negative (TN) procedures divided by the sum of the TN and false-positives (FP) [32-34]. The evaluation of the positive predictive value is achieved by $\mathrm{TP} /(\mathrm{TP}+\mathrm{FP})$, the negative predictive value (NPV) by TN/(TN + FN), whereas accuracy is calculated by the sum of TP and TN divided by the whole population [34].

Data analysis was carried out using the Statistical Pack-age for the Social Sciences software (SPSS, version 17.0) in which the Chi-square test was employed. All statistical measures were considered significant at the 95\% confidence interval $(\mathrm{p}<0.05)$.

\section{Results}

A SN was identified on lymphoscintigraphy as visualization of an accumulation of foci in 52 out of 55 patients (94.5\%) with visualization of a minimum of $1 \mathrm{SN}$ in $69.1 \%(38 / 55)$ of cases and a maximum of 2 SNs in $25.4 \%(14 / 55)$ of patients. During surgery, a number of SNs were successfully isolated in $94.5 \%$ (52/55) of cases with a minimum of $1 \mathrm{SN}$ up to a maximum of 9 SNs. In total, 105 individual SNs were surgically harvested during SLNB in 52 patients and none were excised in the remaining 3 patients. The complete range of sentinel node findings identified per patient is presented in Table 1. In contrast, 424 ALNs ranging between 1 and 25, were removed during partial or total ALND in 55 procedures including the 3 patients where no SNs were visualized and identified (Table 2).

An overview of the findings of this study is depicted in Figure 1. The sensitivity of SLNB in axillary staging was found to be $62.5 \%$, the specificity $84.1 \%$ whereas the PPV and NPV were established as $41.6 \%$ and $92.5 \%$ respectively.

Chi-square test results showed that there is a significant statistical association ( $<0.05)$ between the metastatic status of the SNs and the corresponding nodal status of the ALNs. In fact, the diagnostic accuracy of this study was established in $80.8 \%$ of cases (42/52), however with a $37.5 \%$ false-negative rate (Table 3 ).

\section{Discussion}

The accuracy of this study was consistent with several audit studies (Table 4) which evaluated the efficacy of the SLNB technique by investigating the concordance between the $\mathrm{SN}$ and the respective ALNs status. The accuracy of the SNs to predict axillary status should ideally exceed $90 \%[21,46]$. Nevertheless, the findings of the current local study established a lower rate of accuracy probably due to a number of factors: the SLNB was at its early stages of implementation with the staff still integrating the learning curve; the small sample size and the use of methylene blue only in some and not all of the cases [15].

Table 1. Number of surgically identified sentinel node/s per patient.

\begin{tabular}{cccc}
\hline $\begin{array}{c}\text { No. of surgically } \\
\text { identified SNs }\end{array}$ & $\begin{array}{c}\text { No. of } \\
\text { patients }\end{array}$ & $\begin{array}{c}\text { Total No. } \\
\text { of SNs }\end{array}$ & $\begin{array}{c}\text { Percentage } \\
\text { (\%) }\end{array}$ \\
\hline 0 & 3 & 0 & $55 \%$ \\
1 & 30 & 30 & $54.6 \%$ \\
2 & 10 & 20 & $18.2 \%$ \\
3 & 6 & 18 & $10.9 \%$ \\
4 & 1 & 4 & $1.8 \%$ \\
5 & 1 & 5 & $1.8 \%$ \\
6 & 2 & 12 & $3.6 \%$ \\
7 & 1 & 7 & $1.8 \%$ \\
8 & 0 & 0 & $0 \%$ \\
9 & 1 & 9 & $1.8 \%$ \\
Totals & 55 & 105 & $100 \%$ \\
\hline
\end{tabular}

Key: SN—sentinel node.

Table 2. Number of surgically identified axillary nodes per patient.

\begin{tabular}{cccc}
\hline $\begin{array}{c}\text { No. of surgically } \\
\text { identified ALNs }\end{array}$ & $\begin{array}{c}\text { No. of } \\
\text { patients }\end{array}$ & $\begin{array}{c}\text { Total No. of } \\
\text { ALNs }\end{array}$ & $\begin{array}{c}\text { Percentage } \\
(\%)\end{array}$ \\
\hline 0 & 0 & 0 & 0 \\
1 & 1 & 1 & $1.8 \%$ \\
2 & 5 & 10 & $9.1 \%$ \\
3 & 3 & 9 & $5.5 \%$ \\
4 & 2 & 8 & $3.6 \%$ \\
5 & 6 & 30 & $10.9 \%$ \\
6 & 8 & 48 & $14.5 \%$ \\
7 & 8 & 56 & $14.5 \%$ \\
8 & 4 & 32 & $7.3 \%$ \\
9 & 4 & 36 & $7.3 \%$ \\
10 & 2 & 20 & $3.6 \%$ \\
11 & 3 & 33 & $5.5 \%$ \\
12 & 0 & 0 & 0 \\
13 & 3 & 39 & $5.5 \%$ \\
14 & 1 & 14 & $1.8 \%$ \\
15 & 3 & 46 & $5.5 \%$ \\
16 & 1 & 16 & $1.8 \%$ \\
$17-24$ & 0 & 0 & 0 \\
25 & 1 & 25 & $1.8 \%$ \\
Totals & 55 & 424 & $100 \%$ \\
\hline
\end{tabular}

Key: ALN—Axillary lymph node. 


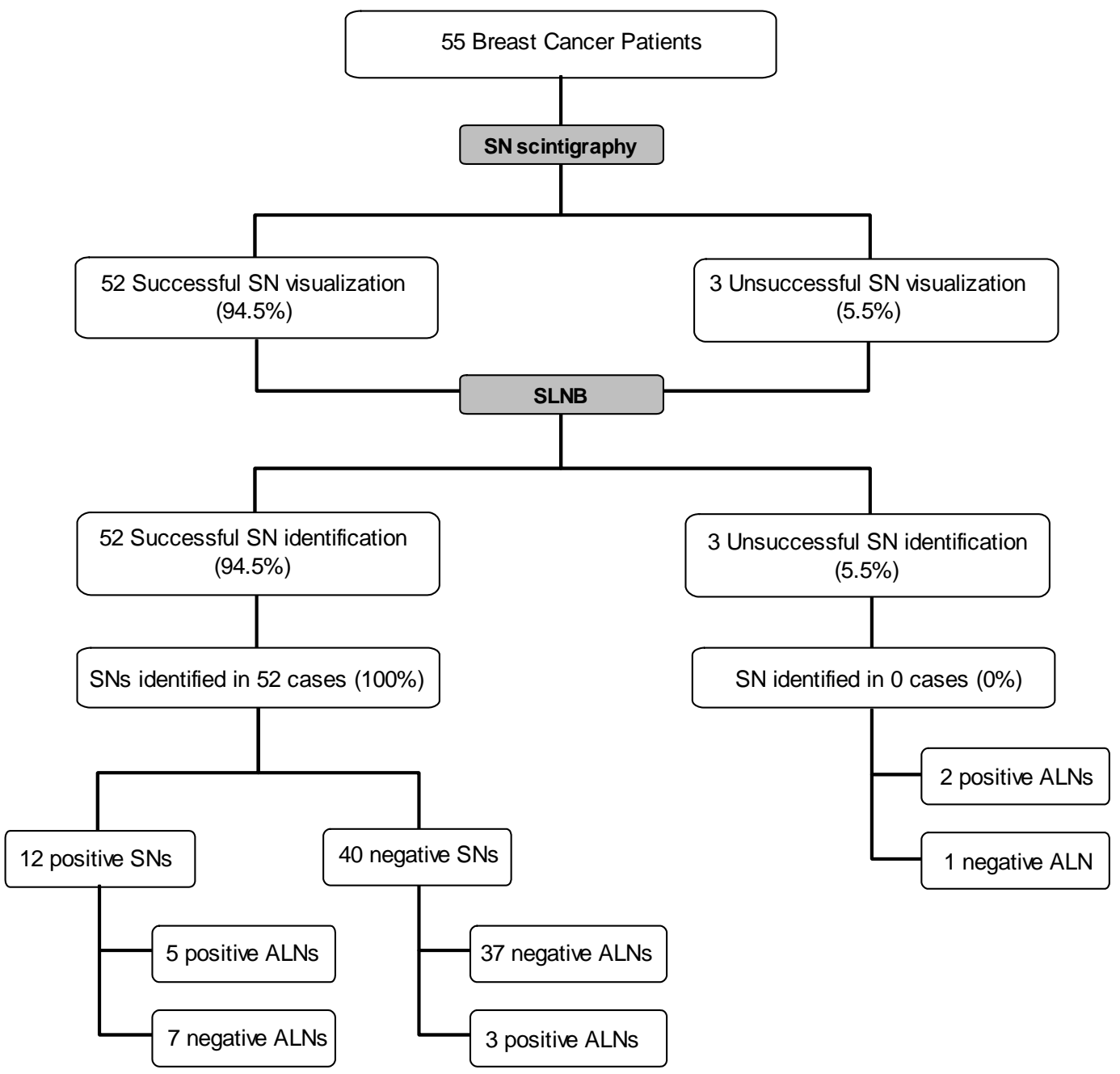

Key: ALN - Axillary lymph node; SLNB - sentinel lymph node biopsy; SN - sentinel node.

Figure 1. Flow chart demonstrating an overview of the findings of the study.

Table 3. Chi-square test showing the relationship between histologic results of SNs and ALNs.

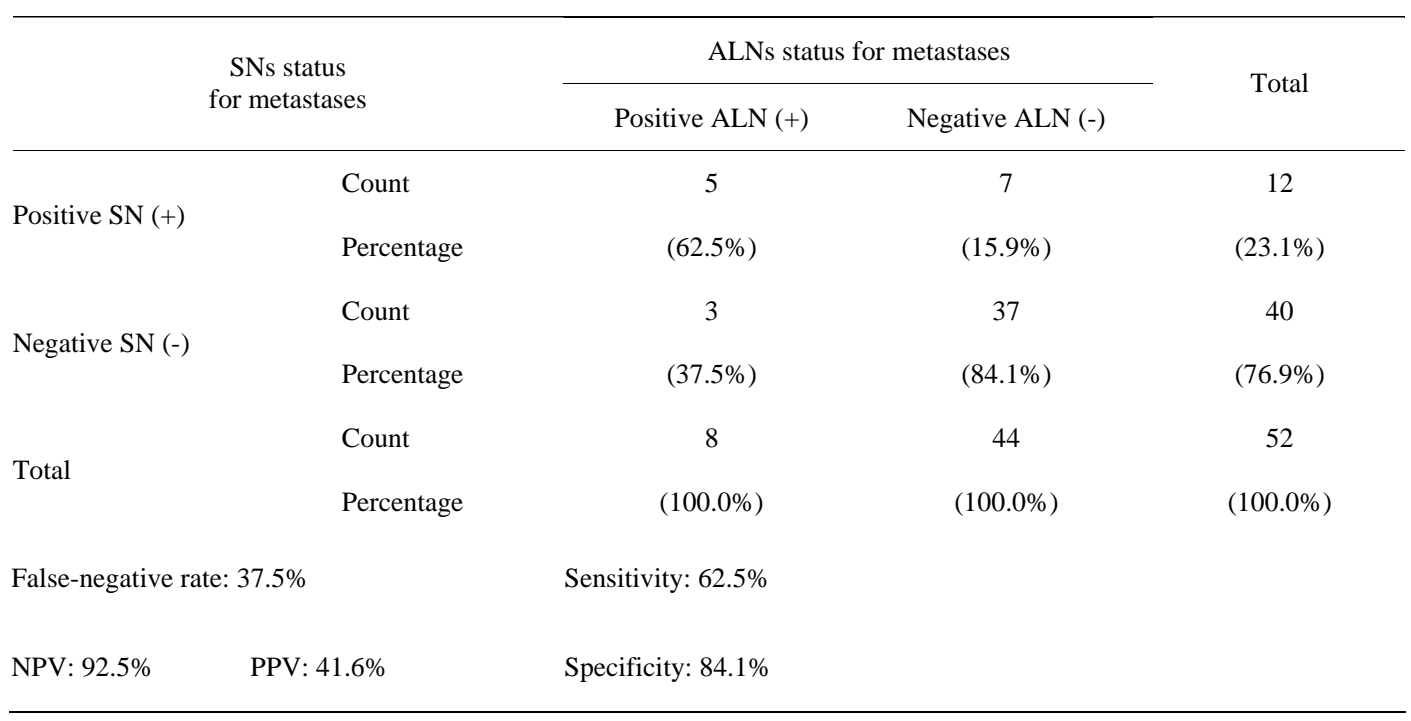

Key: ALN—Axillary lymph node; SN—Sentinel node; NPV—Negative predictive value; PPV—Positive predictive value. 
Table 4. Comparison of the SLNB identification and false-negative rates in other studies.

\begin{tabular}{|c|c|c|c|c|c|c|}
\hline Authors & Year & $\begin{array}{l}\text { Sample } \\
\text { Size }\end{array}$ & Injection Technique & $\begin{array}{l}\text { SN/s Identification } \\
\text { during SLNB }\end{array}$ & $\begin{array}{c}\text { Concordance } \\
\text { between SN } \\
\text { and ALNs }\end{array}$ & FNR \\
\hline De Cicco et al. [24] & 1998 & 250 & Various & $96 \%$ & $97.5 \%$ & $\mathrm{n} / \mathrm{a}$ \\
\hline Hill et al. [35] & 1999 & 500 & Intradermal or Peri-tumoral & $93 \%$ & $\mathrm{n} / \mathrm{a}$ & $10.6 \%$ \\
\hline Borgstein et al. [36] & 2000 & 220 & Peri-tumoral or Peri-areolar & $94 \%$ & $\mathrm{n} / \mathrm{a}$ & $5 \%$ \\
\hline Haigh et al. [37] & 2000 & 283 & Peri-tumoral & $81 \%$ & $98.7 \%$ & $3.2 \%$ \\
\hline Birdwell et al. [38] & 2001 & 136 & Peri-tumoral & $94 \%$ & $\mathrm{n} / \mathrm{a}$ & $8 \%$ \\
\hline Motomura et al. [39] & 2001 & 138 & Subdermal & $94.9 \%$ & $96.5 \%$ & 0 \\
\hline Wong et al. [40] & 2001 & 1436 & Various & $90 \%$ & $\mathrm{n} / \mathrm{a}$ & $8.3 \%$ \\
\hline Kumar et al. [41] & 2003 & 59 & Intradermal or subdermal & $100 \%$ & $\mathrm{n} / \mathrm{a}$ & 0 \\
\hline Chagpar et al. [42] & 2004 & 1431 & Peri-tumoral & $91.1 \%$ & $\mathrm{n} / \mathrm{a}$ & $8.6 \%$ \\
\hline Chagpar et al. [42] & 2004 & 148 & Sub-areolar & $99.3 \%$ & $\mathrm{n} / \mathrm{a}$ & $8.3 \%$ \\
\hline Chagpar et al. [42] & 2004 & 183 & Peri-areolar & $95.6 \%$ & $\mathrm{n} / \mathrm{a}$ & $8.9 \%$ \\
\hline D’Eredita et al. [43] & 2005 & 115 & Subdermal & $94.8 \%$ & $\mathrm{n} / \mathrm{a}$ & $9.1 \%$ \\
\hline D’Eredita et al. [43] & 2005 & 40 & Sub-areolar & $97.5 \%$ & $\mathrm{n} / \mathrm{a}$ & 0 \\
\hline Martin et al. [44] & 2005 & 645 & Dermal or subdermal & $\mathrm{n} / \mathrm{a}$ & $\mathrm{n} / \mathrm{a}$ & $7.4 \%$ \\
\hline Martin et al. [44] & 2005 & 123 & Peri-areolar or subareolar & $\mathrm{n} / \mathrm{a}$ & $\mathrm{n} / \mathrm{a}$ & $7.3 \%$ \\
\hline Martin et al. [44] & 2005 & 478 & Peri-tumoral & $\mathrm{n} / \mathrm{a}$ & $\mathrm{n} / \mathrm{a}$ & $8.6 \%$ \\
\hline Namwongprom et al. [3] & 2005 & 35 & Peri-tumoral or subdermal & $91.4 \%$ & $87.5 \%$ & $30.8 \%$ \\
\hline Povoski et al. [45] & 2007 & 371 & Intraparenchymal Intradermal or Sub-areolar & $95 \%$ & $\mathrm{n} / \mathrm{a}$ & $\mathrm{n} / \mathrm{a}$ \\
\hline Ferreira et al. [46] & 2008 & 50 & Sub-areolar & $96 \%$ & $97.9 \%$ & $6.25 \%$ \\
\hline Abdollahi et al. [47] & 2009 & 202 & Peri-areolar (intra-dermal) & $89 \%$ & $\mathrm{n} / \mathrm{a}$ & $\mathrm{n} / \mathrm{a}$ \\
\hline Nakashima et al. [34] & 2009 & 60 & Peri-areolar (intradermal) & $98.3 \%$ & $71.4 \%$ & $1.7 \%$ \\
\hline This study & 2012 & 55 & Peri-arealor (subdermal) & $94.5 \%$ & $80.8 \%$ & $37.5 \%$ \\
\hline
\end{tabular}

Key: SLNB—Sentinel lymph node biopsy; ALN—Axillary lymph node; SN—Sentinel node; FNR—False negative rate; n/a—not applicable.

These audit studies presented SN identification rates during SLNB ranging from $81 \%$ to $100 \%$, which compare well with the local identification rate. Identification rates are expected to improve as the experience with the biopsy technique continues to evolve, with the procedure becoming more refined thus increasing the accuracy $[3,21,30]$.

For the SLNB to be effective, the "true" SN must be identified, that is, the node that receives direct drainage from the tumor [30]. Successful lymphoscintigraphy, the use of the methylene blue dye technique together with the gamma-probe guided SLNB (combined method) and the absence of metastatically involved SNs and ALNs increase the ability of the intra-operative SN identifica- tion during SLNB $[15,29]$. There appears to be an ongoing debate on the optimal number of sentinel nodes that need to be harvested to ensure accurate axillary staging. Wong et al. [40] argue that the identification of a single SN impacts negatively on the FNR when compared to the removal of multiple SNs which drastically minimizes FNRs. As opposed to the practice of excising all stained and/or radioactive lymph nodes, Lynch et al. [48] insist that $100 \%$ accuracy is achieved when a minimum of 3 SNs are excised. In contrast, the observed decline in the accuracy of the present study and an established high FNR could be attributed to the fact that in half of cases (54.6\%) only a solitary SN was excised with only $21.7 \%$ of patients having 3 or more SNs removed. 
Factors that may compromise diagnostic accuracy involve the choice of radiopharmaceutical, the technique and site of injection and patient positioning during imaging [21,31]. A large multi-centre clinical trial published by Chagpar et al. [42] reported an identification rate of 91.1\% for peritumoral injections, that contrasts with the 99.3\% rate achieved when the patients in the same study underwent sub-areolar injections. Gross tumor involvement of the nodes may also impair the uptake of both the radiopharmaceutical (detected by imaging and/or the gamma probe) and by the blue dye resulting in failure in isolating the true SN [19]. In fact 2 out of the 3 patients $(66.67 \%)$ in the local study who had no SNs surgically identified, were histologically diagnosed with Grade II and Grade III invasive ductal carcinoma respectively. The latter case being reported to harbor ALN metastases in 25 nodes. This metastatic infiltration implies that the failure to harvest the nodes was presumably due to the aforementioned arguments rather than failure of the SLNB technique [49].

Although SLNB has been proposed as an alternative to the routine aggressive ALND, reported FNRs vary widely from $0 \%$ to $30.8 \%$ (Table 4 ). The FNR of the SLNB alternatively referred to as skip metastases, represents the proportion of patients with no SNs harboring metastases but with subsequently positive ALNs for metastases [13, 31]. The implications of FNR are that it unreliably classifies patients as having no metastatic nodes, in the likelihood of persistent nodal involvement [44].

The sensitivity (62.5\%) and specificity (84.1\%) of the local study are similar to the ones obtained by Nakashima and colleagues [34] who report a sensitivity of $62.1 \%$ and specificity of $81.5 \%$. The weakness in this local study is evident by the higher FNR. This unexpected finding is higher than other FNRs reported in the literature [3,8,23-25]. It is well documented that several factors account for false-negative results in SLNB. Tumor cells passing through the SNs and metastasizing into other nodes in the axillary basin metastasizing into other nodes in the axillary basin (non-SNs) may cause FNRs $[3,34]$. Adding to this, an extensive involvement of the relay $\mathrm{SN}$ may obstruct or alter the lymphatic drainage to a node other than the "true" SN, compromising the FNR $[34,50]$. The excision of only $1 \mathrm{SN}$ has also been suggestive of high FNRs, so the trend is to harvest multiple nodes by simultaneously employing both the blue dye and intra-operative gamma probe techniques $[40,44]$. Another mechanism that is responsible for high FNRs is the different pathological analysis of SNs. Immunohistochemistry after $\mathrm{H}$ \& $\mathrm{E}$ staining reveals occult lymph node metastases and significantly reduces the FNR by $10 \%-36 \%[13,51,52]$. Also in some rare cases lymphatic drainage occurs through the mediastinum and not through the axilla [20, 21].
Concern has been raised over the limited technical experience in SLNB which results in a relatively high incidence of FNR $[3,16,47]$ as observed in this local study. In fact, it was noted that $70 \%$ of false-negatives occurred during the biopsy of the first 30 patients performed locally with this technique. False-negatives may arise due to the inability to harvest the true sentinel node during the biopsy, therefore training on how to selectively identify the $\mathrm{SN}$ is recommended. One possible way of achieving this, as suggested by Cantin et al. [53] is to have the personnel attend a continuing medical education course, or alternatively host a visiting surgeon to share the expertise in SLNB. At the end of the training programs and once all entities attain proficiency in the technique, the FNR are expected to improve [44]. Literature documents that a definite technical "learning curve" exists before the personnel become proficient in SLNB because of its technically-demanding process [54]. When the technique is mastered it yields successful biopsies without compromising survival; however the number of cases required accomplishing skill and aptitude is still controversial $[3,20]$. In the interim, owing to the high incidence of FNR experienced locally, it is not advisable to locally abandon ALND until the SN biopsy is locally established to be an accurate staging technique [44].

Another limitation encountered in the local study was the assumption that that the two performing surgeons had acquired the same level of expertise in the SLNB technique which was being introduced locally.

\section{Conclusions}

This study provides a preliminary evaluation of the local introduction of the lymphoscintigraphy guided SLNB as a staging technique. In $80.8 \%$ of cases, the SN accurately predicted the metastatic status of the axilla and thus establishes the usefulness of SLNB. However, the impact of the $37.5 \%$ FNR remains unclear and emphasis should be made to extensively reduce this rate as it can jeopardize patient prognosis since missed nodes might lead to under staging while retained metastatic axillary nodes act as a potential source of distant metastases [1,34,50]. FNRs are regarded as the indicator of the accuracy of SLNB procedure and may relate to the technical factors that ought to improve once the learning curve is bypassed [49,55].

A study using the same methodology and objectives is recommended in a few years' time whereby the "learning curve" of SLNB is expected to improve locally. Once the transitional period of the SLNB as an alternative to ALND takes place, further investigation concerning the sensitivity, specificity, accuracy and false-negative rates of SLNB are warranted within the local scenario. In addition, when SLNB is established as the standard treatment of care for sentinel node-negative patients, a pro- 
spective study designed to follow-up patients for the possible recurrence of metastases and survival outcome should be conducted [56] since limited data is available in this context locally.

With the introduction of the SN concept, breast cancer management has dramatically evolved towards minimally invasive approaches [1,57]. The intent of this developing treatment paradigm is to maximize the concordance rates whilst minimizing the FNRs. Hopefully, in the near future, improvements in the SLNB technique should increase its usefulness as a staging technique and would obviate the need of ALND in patients with a negative SN status for metastases, thus improving the prognosis of Maltese breast cancer patients.

\section{REFERENCES}

[1] A. Chakravorty, N. Sanmugalingam, A. Shrestha, et al., "Axillary Nodal Yields: A Comparison between Primary Clearance and Completion Clearance after Sentinel Lymph Node Biopsy in the Management of Breast Cancer," European Journal of Surgical Oncology, Vol. 37, No. 2, 2011, pp. 122-126. doi:10.1016/j.ejso.2010.10.005

[2] E. A. Newman and L. A. Newman, "Lymphatic Mapping Techniques and Sentinel Lymph Node Biopsy in Breast Cancer," Surgical Clinics of North America, Vol. 87, No. 2, 2007, pp. 353-364. doi:10.1016/j.suc.2007.01.013

[3] S. Namwongprom, S. Boonyaprapa, M. Ekmahachai, et al., "Breast Lymphoscintigraphy for Sentinel Node Identification in Breast Cancers with Clinically-Negative Axillary Nodes,” Singapore Medical Journal, Vol. 46, No. 12, 2005, pp. 688-692.

[4] X. Sun, J.-J. Liu, Y.-S. Wang, et al., "Roles of Preoperative Lymphoscintigraphy for Sentinel Lymph Node Biopsy in Breast Cancer Patients," Japanese Journal of Clinical Oncology, Vol. 40, No. 8, 2010, pp. 722-725. doi:10.1093/jjco/hyq052

[5] M. R. S. Keshtgar, W. A. Waddington, S. R. Lakhani, et al., "The Sentinel Node in Surgical Oncology," SpringerVerlag, Berlin, 1999, pp. 49-89.

[6] S. Pesek, T. Ashikaga, L. E. Krag, et al., "The FalseNegative Rate of Sentinel Node Biopsy in Patients with Breast Cancer: A Meta-Analysis,” World Journal of Surgery, Vol. 36, No. 9, 2012, pp. 2239-2251. doi:10.1007/s00268-012-1623-z

[7] B. Duchaj, P. Chvalny, J. Vesely, et al., "Radionavigated Detection of Sentinel Nodes in Breast Carcinoma-First Experiences of Our Department,” Bratislavské Lekárske Listy, Vol. 111, No. 1, 2010, pp. 33-37.

[8] I. Bokhari, Q. Tahir, S. Rasul, et al., "Early Experience of Sentinel Lymph Node Biopsy in Breast Cancer,” Journal of Surgery Pakistan International, Vol. 14, No. 1, 2009, pp. 29-32.

[9] V. Vijayakumar, P. S. Boerner, A. B. Jani, et al., “A Critical Review of Variables Affecting the Accuracy and False-Negative Rate of Sentinel Node Biopsy Procedures in Early Breast Cancer," Nuclear Medicine Communica- tions, Vol. 26, 2005, pp. 395-405. doi:10.1097/00006231-200505000-00002

[10] G. Pechlivanides, D. Vassilaros, A. Tsimpanis, et al., "Sentinel Node Biopsy for Breast Cancer Patients: Issues for Discussion and Our Practice,” Pathology Research International, Vol. 2011, 2011, Article ID: 109712. doi:10.4061/2011/109712

[11] R. E. Mansel, L. Fallowfield, M. Kissin, et al., "Randomized Multicenter Trial of Sentinel Node Biopsy versus Standard Axillary Treatment in Operable Breast Cancer: The ALMANAC Trial,” Journal of National Cancer Institute, Vol. 98, No. 9, 2006, pp. 599-609. doi:10.1093/jnci/djj158

[12] W. E. Burak Jr., M. J. Walker, L. D. Yee, et al., "Routine Preoperative Lymphoscintigraphy Is Not Necessary Prior to Sentinel Node Biopsy for Breast Cancer,” The American Journal of Surgery, Vol. 177, No. 6, 1999, pp. 445449. doi:10.1016/S0002-9610(99)00088-4

[13] J. Leikola, "Sentinel Lymph Node Biopsy as a Diagnostic Tool in the Treatment of Breast Cancer," Academic Dissertation, Helsinki, 2008, pp. 11-67.

https://helda.helsinki.fi/bitstream/handle/10138/22788/se ntinel.pdf?sequence $=1$

[14] European Commission, "Eurostat: Causes of Death Statistics-Statistics Explained,” 2012.

http://epp.eurostat.ec.europa.eu/statistics_explained/index .php/Causes_of_death_statistics

[15] G. Camilleri, K. B. Grima and F. Zarb, "Correlation of Number and Identification of Sentinel Nodes during Radiographer Led Lymphoscintigraphy Prior to Sentinel Lymph Node Biopsy in Breast Cancer Patients,” Radiography, Vol. 18, No. 1, 2011, pp. 9-14. doi:10.1016/j.radi.2011.11.003

[16] S. Zakaria, A. C. Degnim, C. G. Kleer, et al., "Sentinel Lymph Node Biopsy for Breast Cancer: How Many Nodes Are Enough?” Journal of Surgical Oncology, Vol. 96, No. 7, 2007, pp. 554-559. doi:10.1002/jso.20878

[17] J. Buscombe, G. Paganelli, Z. E. Burak, et al., "Sentinel Node in Breast Cancer Procedural Guidelines,” European Journal of Nuclear Medicine and Molecular Imaging, Vol. 34, No. 12, 2007, pp. 2154-2159. doi:10.1007/s00259-007-0614-Z

[18] C. D. Collins, "The Sentinel Node in Breast Cancer: An Update,” Cancer Imaging, Vol. 5, No. A, 2005, pp. S3-S9. doi:10.1102/1470-7330.2005.0039

[19] O. E. Nieweg, P. J. Tanis and B. B. R. Kroon, "The Definition of a Sentinel Node," European Journal of Nuclear Medicine and Molecular Imaging, Vol. 8, No. 6, 2001, pp. 538-541. doi:10.1007/s10434-001-0538-y

[20] A. Goyal, R. G. Newcombe, R. E. Mansel, et al., "Role of Routine Preoperative Lymphoscintigraphy in Sentinel Node Biopsy for Breast Cancer,” European Journal of Cancer, Vol. 41, No. 2, 2005, pp. 238-243. doi:10.1016/j.ejca.2004.05.008

[21] H. Rempp, "Sentinel Lymph-Node Radiodetection in Breast Cancer: Results of a Clinical Study and Development of a Quantitative Approach,” Thesis, University of Nice Sophia Antipolis, Nice, 2003, pp. 3-83. 
[22] K. M. McMasters, S. L. Wong, T. M. Tuttle, et al., "Preoperative Lymphoscintigraphy for Breast Cancer Does Not Improve the Ability to Identify Axillary Sentinel Lymph Nodes,” Annals of Surgery, Vol. 231, No. 5, 2000, pp. 724-731. doi:10.1097/00000658-200005000-00013

[23] F. Marchal, P. Rauch, O. Morel, et al., "Results of Preoperative Lymphoscintigraphy for Breast Cancer Are Predictive of Identification of Axillary Sentinel Lymph Nodes,” World Journal of Surgery, Vol. 30, No. 1, 2006, pp. 55-62. doi:10.1007/s00268-005-0145-3

[24] C. De Cicco, M. Cremonesi, A. Luini, et al., "Lymphoscintigraphy and Radioguided Biopsy of the Sentinel Axillary Node in Breast Cancer," Journal of Nuclear Medicine, Vol. 39, No. 12, 1998, pp. 2080-2084.

[25] A. E. Giuliano, D. M. Kirgan, J. M. Guenther, et al., "Lymphatic Mapping and Sentinel Lymphadenectomy for Breast Cancer,” Annals of Surgery, Vol. 220, No. 3, 1994, pp. 391-398. doi:10.1097/00000658-199409000-00015

[26] G. M. Filippakis and G. Zografos, "Contraindications of Sentinel Lymph Node Biopsy: Are There Any Really?" World Journal of Surgical Oncology, Vol. 5, No. 29, 2007, p. 10. doi:10.1186/1477-7819-5-10

[27] B. R. Krynyckyi, S. C. Kim and C. K. Kim, "Preoperative Lymphoscintigraphy and Triangulated Patient Body Marking Are Important Parts of the Sentinel Node Process in Breast Cancer,” World Journal of Surgical Oncology, Vol. 3, 2005, p. 56. doi:10.1186/1477-7819-3-56

[28] A. H. Chakera, B. Hesse, Z. Burak, et al., "EANMEORTC General Recommendations for Sentinel Node Diagnostics in Melanoma," European Journal of Nuclear Medicine and Molecular Imaging, Vol. 36, No. 10, 2009, pp. 1713-1742. doi:10.1007/s00259-009-1228-4

[29] M. E. Straver, P. Meijnen, G. van Tienhoven, et al., "Sentinel Node Identification Rate and Nodal Involvement in the EORTC 10981-22023 AMAROS Trial,” Annals of Surgical Oncology, Vol. 17, No. 7, 2010, pp. 1854-1861. doi:10.1245/s10434-010-0945-Z

[30] Department of Health and Ageing, "Sentinel Lymph Node Biopsy in Breast Cancer: MSAC Reference 1065 Assessment Report," Commonwealth of Australian Government Publishing Service, Canberra, 2006.

http://www.msac.gov.au/internet/msac/publishing.nsf/Co ntent/BCDC2A9D05A33761CA2575AD0082FD32/\$File /1065\%20-\%20Sentinel\%20Lymph\%20Node\%20Biopsy \%20Report.pdf

[31] M. H. E. Doting, "Sentinel Lymph Node Biopsy in Breast Cancer and Melanoma," Thesis, University Library, Groningen, 2007, pp. 43-82.

[32] J. Bonnema and C. J. H. van de Velde, "Sentinel Lymph Node Biopsy in Breast Cancer,” Annals of Oncology, Vol. 13, No. 10, 2002, pp. 1531-1537. doi:10.1093/annonc/mdf319

[33] A. G. Lalkhen and A. McCluskey, "Clinical Tests: Sensitivity and Specificity," Continuing Education in Anaesthesia, Critical Care \& Pain, Vol. 8, No. 6, 2008, pp. 221-223. doi:10.1093/bjaceaccp/mkn041

[34] K. Nakashima, J. Kurebayashi, H. Sonoo, et al., "Preoperative Dynamic Lymphoscintigraphy Predicts Sentinel Lymph Node Metastasis in Patients with Early Breast
Cancer,” Breast Cancer, Vol. 17, No. 1, 2010, pp. 17-21. doi:10.1007/s12282-009-0123-y

[35] A. D. K. Hill, K. N. Tran, T. Akhurst, et al., "Lessons Learned from 500 Cases of Lymphatic Mapping for Breast Cancer," Annals of Surgery, Vol. 229, No. 4, 1999, pp. 528-535. doi:10.1097/00000658-199904000-00012

[36] P. J. Borgstein, S. Meijer, R. J. Pijpers, et al., "Functional Lymphatic Anatomy for Sentinel Node Biopsy in Breast Cancer: Echoes from the Past and the Periareolar Blue Method," Annals of Surgery, Vol. 232, No. 1, 2000, pp. 81-89. doi:10.1097/00000658-200007000-00012

[37] P. I. Haigh, N. M. Hansen, K. Qi, et al., "Biopsy Method and Excision Volume Do Not Affect Success Rate of Subsequent Sentinel Lymph Node Dissection in Breast Cancer," Annals of Surgical Oncology, Vol. 7, No. 1, 2000, pp. 21-27. doi:10.1007/s10434-000-0021-1

[38] R. L. Birdwell, K. L.Smith, B. J. Betts, et al., "Breast Cancer: Variables Affecting Sentinel Lymph Node Visualization at Preoperative Lymphoscintigraphy,” Radiology, Vol. 220, No. 1, 2001, pp. 47-53.

[39] K. Motomura, H. Inaji, Y. Komoike, et al., "Combination Technique is Superior to Dye Alone in Identification of the Sentinel Node in Breast Cancer Patients," Journal of Surgical Oncology, Vol. 76, No. 2, 2001, pp. 95-99. doi:10.1002/1096-9098(200102)76:2<95::AID-JSO1018> 3.0.CO;2-D

[40] S. L. Wong, M. J. Edwards, C. Chao, et al., "Sentinel Lymph Node Biopsy for Breast Cancer: Impact of the Number of Sentinel Nodes Removed on the False-Negative Rate," Journal of the American College of Surgeons, Vol. 192, No. 6, 2001, pp. 684-691. doi:10.1016/S1072-7515(01)00858-4

[41] R. Kumar, S. Jana, S. I. Heiba, et al., "Retrospective analysis of Sentinel Node Localization in Multifocal, Multicentric, Palpable, or Nonpalpable Breast Cancer," Journal of Nuclear Medicine, Vol. 44, No. 1, 2003, pp. 7-10.

[42] A. Chagpar, R. C. Martin 3rd, C. Chao, et al., "Validation of Subareolar and Periareolar Injection Techniques for Breast Sentinel Lymph Node Biopsy," Archives of Surgery, Vol. 139, No. 6, 2004, pp. 614-620. doi:10.1001/archsurg.139.6.614

[43] G. D’Eredita, C. Giardina, A. M. Guerrieri and T. Berardi, "A Further Validation of Subareolar Injection Technique for Breast Sentinel Lymph-Node Biopsy,” Annals of Surgical Oncology, Vol. 13, No. 5, 2006, pp. 701-717. doi:10.1245/ASO.2006.04.027

[44] R. C. G. Martin 2nd, A. Chagpar, C. R. Scoggins, et al., "Clinicopathologic Factors Associated with False-Negative Sentinel Lymph-Node Biopsy in Breast Cancer," Annals of Surgery, Vol. 241, No. 6, 2005, pp. 1005-1015. doi:10.1097/01.sla.0000165200.32722.02

[45] S. P. Povoski, D. C. Young, M. J. Walker, et al., "Re-Emphasizing the Concept of Adequacy of Intraoperative Assessment of the Axillary Sentinel Lymph Nodes for Identifying Nodal Positivity during Breast Cancer Surgery," World Journal of Surgical Oncology, Vol. 5, 2007, p. 18. doi:10.1186/1477-7819-5-18

[46] Ferreira P, Baía R, António A, et al., “Sentinel lymph 
Node Biopsy: Technique Validation at the Setúbal Medical Centre, Portugal,” Ecancer, Vol. 3, 2009, p.124.

[47] A. Abdollahi, A. Jangjoo, Dabbagh Kakhki VR, et al., "Factors Affecting Sentinel Lymph Node Detection Failure in Breast Cancer Patients Using Intradermal Injection of the Tracer," Revista Española de Medicina Nuclear, Vol. 29, No. 2, 2010, pp. 73-77. doi:10.1016/j.remn.2009.10.001

[48] M. A. Lynch, J. Jackson, J. A. Kim, et al., "Optimal Number of Radioactive Sentinel Lymph Nodes to Remove for Accurate Axillary Staging of Breast Cancer,” Surgery, Vol. 144, No. 4, 2008, pp. 525-532. doi:10.1016/j.surg.2008.06.005

[49] J. M. East, C. S. P.Valentine, E. Kanchev, et al., "Sentinel Lymph Node Biopsy for Breast Cancer Using Methylene Blue Dye Manifests a Short Learning Curve among Experienced Surgeons: A Prospective Tabular Cumulative Sum (CUSUM) Analysis,” BMC Surgery, 2009, Vol. 9, No., p. 2.doi:10.1186/1471-2482-9-2

[50] E. Hindié, D. Groheux, I. Brenot-Rossi, et al., "The Sentinel Node Procedure in Breast Cancer: Nuclear Medicine as the Starting Point,” Journal of Nuclear Medicine, Vol. 52, No. 3, 2011, pp. 405-414. doi:10.2967/jnumed.110.081711

[51] K. Motomura, C. Egawa, Y. Komoike, et al., "Validation and Controversy of Sentinel Node Biopsy in Breast Cancer,” Journal of Breast Cancer, Vol. 9, No. 2, 2006, pp. 74-83. doi:10.4048/jbc.2006.9.2.74
[52] G. Cserni, S. Bianchi, V. Vezzosi, et al., "The Value of Cytokeratin Immunohistochemistry in the Evaluation of Axillary Sentinel Lymph Nodes in Patients with Lobular Breast Carcinoma,” Journal of Clinical Pathology, Vol. 59, No. 5, 2006, pp. 518-522. doi:10.1136/jcp.2005.029991

[53] J. Cantin, H. Scarth, M. Levine, et al., "Clinical Practice Guidelines for the Care and Treatment of Breast Cancer: 13. Sentinel Lymph Node Biopsy," Canadian Medical Association Journal, Vol. 165, No. 2, 2001, pp. 166-173.

[54] G. Mariani, L. Moresco, G. Viale, et al., "Radioguided Sentinel Lymph Node Biopsy in Breast Cancer Surgery," Journal of Nuclear Medicine, Vol. 42, No. 8, 2001, pp. 1198-1215.

[55] T. Kim, A. E. Giuliano and G. H. Lyman, "Lymphatic Mapping and Sentinel Lymph Node Biopsy in EarlyStage Breast Carcinoma: A Metaanalysis,” ACS, Vol. 106, No. 1, 2005, pp. 4-16.

[56] B. van der Vegt, M. H. Doting, P. L. Jager, et al., "Axillary Recurrence after Sentinel Lymph Node Biopsy," European Journal of Surgical Oncology, Vol. 30, No. 7, 2004, pp. 715-720. doi:10.1016/j.ejso.2004.05.015

[57] G. Vlastos and H. M. Verkooijen, "Minimally Invasive Approaches for Diagnosis and Treatment of Early-Stage Breast Cancer,” The Oncologist, Vol. 12, No. 1, 2007, pp. 1-10. doi:10.1634/theoncologist.12-1-1 